\title{
正则图迭线图的 1-因子分解
}

\author{
赵 光 复 \\ (北京理工大学应用数学系, 北京 100081 )
}

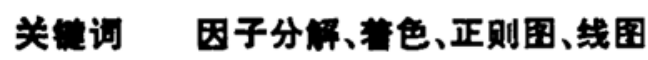

哪些图可 1-因子分解？换言之, 哪些图是正则 1 类图? 这是一个尚未解决的有 诹问 题 ${ }^{[1,2]}$. 众所周知, 四色定理成立的一个充分必要条件是每个无桥的 3-正则平面图可 1-因子 分解 ${ }^{(3)}$. 由此可以看出上述问题的意义和难度. Jaeger ${ }^{[4]}$ 证明, 若一个有偶数条线的图可 1因子分解, 则其线图也可 1-因子分解. 这就是说, 线图变换对图的可 1-因子分解性具有某种 程度的传递作用. 本文的主要结果是

定理 1 若图 $G$ 可 1-因子分解, 则对任意 $k \geqslant 2$, 其 $k$ 次迭线图 $L^{k}(G)$ 可 1-因子分 解.

定理 2 若图 $G$ 可 1-因子分解, 则对任意 $k \geqslant 1$, 其 $k$ 次迭线图 $L^{k}(G)$ 可 1-因子分 解当且仅当 $L^{k}(G)$ 有偶数个点.

这些定理表明, 线图变换对图的可 1-因子分解性还具有某种程度隔代传递作用. 文中采 用的记号和术语凡未加说明的均见文献 [3].

记 $G$ 是一个有 $n$ 个点的 $d$ 度正则图, $n_{k}, d_{k}$ 分别表示 $L^{k}(G)$ 的点数和度. 由

$$
d_{k}-2\left(d_{k-1}-1\right), n_{k}-\frac{1}{2} n_{k} d_{k}
$$

及文献 [4],有

$$
\text { 引理 } 1 \quad n_{k}=n \prod_{i=0}^{k-1}\left(2^{t-1} d-2^{t}+1\right), d_{k}=2^{k}(d-2)+2 .
$$

引理 2 设图 $G$ 可 1 -因子分解. 若 $n_{1}$ 为偶数, 则对任意 $k \geqslant 1, n_{k}$ 为偶数, 从而 $L^{k}(G)$ 可 1-因子分解; 若 $n_{1}$ 为奇数, 则对任意 $k \geqslant 2, n_{k}$ 为偶数, 从而 $L^{k}(G)$ 可 1-因子分解当且 仅当 $L^{2}(G)$ 可 1-因子分解.

由此, 为了证明上述定理, 我们只需证明, 当 $G$ 是奇度正则可 1-因子分解图时, $L^{2}(G)$ 可 1-因子分解.

设 $F=\bigcup_{i=1}^{d} F_{s}$ 是 $G$ 的一个 1-因子分解, $d-2 b+1, C-\left\{c_{1}, c_{2}, \cdots, c_{d}\right\}$ 是色集. 则 $\sigma: e \rightarrow c_{s}, e \in F$, 是 $G$ 的 $d$ 线着色. 考虑以 $C$ 为点集的完全图 $K_{d}$. 记

$$
Z_{r}=c_{d} c_{r} c_{r-1} c_{r+1} \cdots c_{r-b+1} c_{r+b-1} c_{r-b} c_{d}, r-1,2, \cdots, b
$$

其中下标除 $d$ 外均取为 $1,2 \cdots 2 b(\bmod 2 b)$. 则 $Z=\bigcup_{-1}^{b} Z_{r}$ 为 $K_{d}$ 的一个 Hamilton 分解.

1992-01-27 收稿

- 国家自然科学䔄金资助项目 
对每个 $Z_{r}$ 按上述顺序定向, 得有向 Hamilton 圈 $\vec{Z}_{r}-c_{r 1} c_{r 2} \cdots c_{r d} c_{r 1}$, 从而得到 $K_{d}$ 的一个 定向 $\vec{K}_{\mathbf{d}}$.

用 $u_{1}, u_{2}, \cdots, u_{*}$ 标定 $G$ 的各点, $S_{i}$ 表示 $G$ 中点 $u_{i}$ 处的星形图. 记

$$
A_{i}-L\left(S_{i}\right), B_{i}-L^{2}\left(S_{i}\right) \text {. }
$$

则 $\left\{A_{i}\right\},\left\{B_{i}\right\}$ 分别为 $L(G), L^{2}(G)$ 的 $K$-划分和 $C$-划分田.

设 $\tau$ 为由 $\sigma$ 导出的 $L(G)$ 的点着色, $\tau_{i}$ 表示 $\tau$ 在 $A_{i}$ 上的限制. 则 $\tau_{i}$ 是 $A_{i}$ 到 $K_{d}$ 的同 构映射. 利用 $\vec{K}_{d}$ 可给出 $A_{i}$ 的定向 $\vec{A}_{i}$, 进而给出 $L(G)$ 的定向 $\overrightarrow{L(G)}$. 记 $v_{r t}^{i}-\tau_{i}^{-1}\left(c_{r t}\right)$, $Z_{r}^{i}-\tau_{i}^{-1}\left(Z_{r}\right), Z_{r}^{i}$ 为 $Z_{r}^{i}$ 相应的定向. 则 $\bigcup_{r} Z_{r}^{i}$ 和 $\bigcup_{r} \vec{Z}_{r}^{i}$ 分别为 $A_{i}, \vec{A}_{i}$ 的 Hamilton 分解和

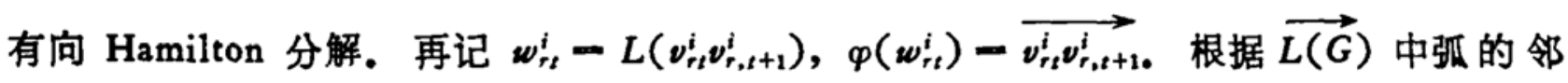
接关系,我们可以对 $E-E\left(L^{2}(G)\right)$ 做如下分类:

$$
\begin{aligned}
& E^{+1}-\left\{w w^{\prime} \in E \mid \varphi(w), \varphi\left(w^{\prime}\right) \text { 有公共终点 }\right\}, \\
& E^{-1}-\left\{w w^{\prime} \in E \mid \varphi(w), \varphi\left(w^{\prime}\right) \text { 有公共始点 }\right\}, \\
& E^{0}-E-E^{ \pm 1} .
\end{aligned}
$$

记 $\Delta_{r}^{i}-\left\{w_{r l}^{i} \mid t-1,2, \cdots, d\right\}, \Delta_{r}-\bigcup_{i} \Delta_{r}^{i}$ 则 $\bigcup_{r} \Delta_{r}^{i}$ 和 $\bigcup_{r} \Delta_{r}$ 分别为 $V\left(B_{i}\right)$ 和 $V-V\left(L^{2}(G)\right)$ 的划分. 用 $H_{r}^{i}$ 表示由线集 $\left\{w w^{\prime} \in E \mid w \in \Delta_{i}^{i}, w^{\prime} \in \Delta_{i}^{i}\right\}$ 导出的 $L^{2}(G)$ 的 子图,并记

这里 $\delta \in\{0, \pm 1\}$.

$$
\begin{array}{cl}
B-\bigcup_{i} B_{i}, & \bar{B}-L^{2}(G)-E(B), \\
H_{r s}-\bigcup_{i, i} H_{r s}^{i !}, & H_{r s}^{s}-H_{r s} \cap E^{\delta}, \\
B_{r s}-B \cap H_{r s}, & B_{r_{r}}^{s}-B_{r s} \cap E^{\delta}, \\
\bar{B}_{r s}-\bar{B} \cap H_{r s}, & \widetilde{B}_{r s}-\bar{B}_{r s} \cap E^{\delta},
\end{array}
$$

给定 $w \in \Delta^{i}, 1 \leqslant s \leqslant b$. 由于 $\vec{Z}_{i}^{i}, \vec{Z}_{i}^{i}$ 均为 $\vec{A}_{i}$ 的有向 Hamilton 圈, 存在唯一的点对 $w^{\prime}, w^{\prime \prime} \in \Delta_{i}^{i}$, 使弧 $\varphi(w)$ 与 $\varphi\left(w^{\prime}\right), \varphi\left(w^{\prime \prime}\right)$ 与 $\varphi(w)$ 分别首尾相邻. 点 $w^{\prime}, w^{\prime \prime}$ 分别称 为 $w$ 在 $B_{r,}^{0}$ 中的外、内邻点, 记作 $N_{r j}^{ \pm 0}(w)$. 线 $w w^{\prime}, w w^{\prime \prime}$ 分别称为 $w$ 在 $B_{r,}^{0}$ 中的外、内邻线, 记作 $\eta_{r i}^{ \pm 0}(w)$. 当 $r \neq s, \delta- \pm 1$ 时, $w$ 在 $B_{r,}^{\delta}$ 中唯一的邻点 $w^{\delta}$ 称为 $w$ 在 $B_{r,}^{\delta}$ 中的共轭点, 记 作 $C_{r,}^{\delta}(w)$. 线 $w w^{\delta}$ 称为 $w$ 在 $B_{r}^{\delta}$ 中的共轭线, 记作 $\theta_{r}^{\delta}(w)$. 类似地, 我们还可以定义 $w$ 在 $\bar{B}_{r}^{0}$ 中的外、内邻点 $\bar{N}_{r i}^{ \pm 0}(w)$, 外、内邻线 $\eta_{r i}^{ \pm 0}(w)$, 以及 $w$ 在 $\bar{B}_{r s}^{o}$ 中的共轭点 $\bar{C}_{r i}^{o}(w)$, 共轭线 $\theta_{r v}^{s}(w)$, 这时允许 $r-s$. 记

$$
\begin{aligned}
& B_{r d}^{ \pm 0}-\left\{\eta_{r, j}^{ \pm 0}(w) \mid w \in \Delta_{r}\right\}, \bar{B}_{r d}^{ \pm 0}-\left\{\eta_{r s}^{ \pm 0}(w) \mid w \in \Delta_{r}\right\}, \\
& B_{r}^{*}-\left(B_{r r}^{0}-\left(\bigcup_{i} \eta_{r r}^{+0}\left(w_{r d}^{i}\right)\right)\right) \cup\left(\bigcup_{r=1 ., d} \bar{\theta}_{r r}^{+1}\left(w_{r t}^{i}\right)\right), \\
& \bar{B}_{r}^{*}-\left(\bar{B}_{r r}^{0}-\left(\bigcup_{i} \eta_{r r}^{+0}\left(w_{r d}^{i}\right)\right)\right) \cup\left(\bigcup_{r=1, d} \bar{\theta}_{r r}^{-1}\left(w_{r t}^{i}\right)\right), \\
& D_{r}^{+1}-\left(\bar{B}_{r r}^{+1}-\left(\bigcup_{r=1, d} \bar{\theta}_{r r}^{+1}\left(w_{r t}^{i}\right)\right)\right) \cup\left(\bigcup_{i} \eta_{r r}^{+0}\left(w_{r d}^{i d}\right)\right),
\end{aligned}
$$




$$
D_{r}^{-1}-\left(\bar{B}_{r r}^{-1}-\left(\bigcup_{r=1, d} \bar{\theta}_{r r}^{-1}\left(w_{r i}^{i}\right)\right)\right) \cup\left(\bigcup_{i} \eta_{r r}^{+0}\left(w_{r d}^{i}\right)\right)
$$

则有

引理 3 若 $r-s$, 则 $B_{r s}-B_{r s}^{0}$ 是 2 正则的; 若 $r \neq s$, 则 $B_{r s}$ 是 4 正则的, 且 $B_{r}^{ \pm 0} \cup B_{r r}^{ \pm 1}$ 是 $B_{r s}$ 的 1-因子分解.

引理 $4 \bar{B}_{r}$ 是 4 正则的, 它可分解为 2-因子, $\bar{B}_{r s}^{0}$ 和两个 1-因子 $\bar{B}_{n}^{ \pm}$. 此外, 当 $r \neq s$ 时, $\bar{B}_{r}^{0}$ 还可分解为两个 1-因子.

引理 $5 D_{r}^{ \pm 1}$ 是 $H_{r r}$ 的两个 1-因子. $B_{r}^{*}, \vec{B}_{r}^{*}$ 是 $H_{r r}$ 的两个 2 -因子, 且分别可分解为两个 1-因子.

现在考虑以 $M-\{1,2, \cdots, b\}$ 为点集的完全图 $K_{b}$, 并根据 $b$ 的奇偶珄分别进行讨论.

情形 $1 . b=2 a$. 设 $I-\bigcup_{a-1}^{b-1} I_{a}$ 为 $K_{b}$ 的一个 1 -因子分解. 记

$$
X_{a}-\bigcup_{r \in I_{a}} B_{r s}, X-\bigcup_{a} X_{a}, Y_{a}-\bigcup_{r \in l_{a}} \bar{B}_{r,}, Y-\bigcup_{a} Y_{a}, W-\bigcup_{r}\left(B_{r}^{*} \cup \bar{B}_{r}^{*} \cup D_{r}^{ \pm 1}\right) .
$$

由引理 3、4, $X_{a}, Y_{a}$ 均可分解为 4 个 1-因子, 从而 $X, Y$ 分别可分解为 $L^{2}(G)$ 的 $4(b-1)$ 个 1-因子. 再由引理 5, $W$ 可分解为 6 个 1-因子. 这样得到 $L^{2}(G)$ 的一个 1-因子分解.

情形 2. $b=2 a+1$. 设 $K_{b+1}$ 是以 $M \cup\{0\}$ 为点集的完全图, $J-\bigcup_{-1}^{b} J_{a}$ 为 $K_{b+1}$ 的一 个 1-因子分解, 其中 $0 \alpha \in J_{a}$. 记 $J_{a}^{*}-J_{\alpha}-\{0 \alpha\}$. 则 $J_{a}^{*}$ 覆盖 $K_{b}$ 中除 $\alpha$ 外的所有点, 即为 $K_{b}$ 的一个拟 1-因子, 而 $J^{*}-\bigcup_{a=1}^{b} J_{a}^{*}$ 为 $K_{b}$ 的一个拟 1-因子分解. 由引理 3、5,

$$
X_{a}-\left(\bigcup_{r \in J_{a}^{*}} B_{r}\right) \cup\left(B_{a}^{*} \cup \bar{B}_{a}^{*}\right)
$$

可分解为 4 个 1-因子. 这样, 我们得到 $L^{2}(G)$ 的 $4 b$ 个 1-因子.

取 $K_{b}$ 的一个 Hamilton 分解 $C-\bigcup_{a-1}^{\infty} C_{a}$, 其中

$$
C_{a}-b, \alpha, \alpha-1, \alpha+1, \cdots, \alpha-a+1, \alpha+a-1, \alpha-a, b,
$$

这里的元除 $b$ 外均取 $1,2, \cdots 2 a(\bmod 2 a)$. 按上述顺序定向得 $\vec{C}_{a}$. 当 $-2 a^{\prime}$ 时, 取

$$
\vec{e}_{\alpha}-\left(\alpha-a^{\prime}, \alpha+a^{\prime}\right),
$$

当 $a-2 a^{\prime}+1$ 时, 取 $\vec{e}_{\alpha}-\left(\alpha+a^{\prime}, \alpha-a^{\prime}+1\right)$. 记

$$
\begin{aligned}
& \vec{P}_{a}-\vec{C}_{a}-\vec{e}_{a}-r_{a 1} r_{a 2} \cdots r_{a b}, \quad Y_{a}^{ \pm 1}-\bigcup_{r s \in C_{\alpha}} \bar{\theta}_{r i}^{ \pm 1}\left(w_{r l}^{i}\right) \text {, }
\end{aligned}
$$

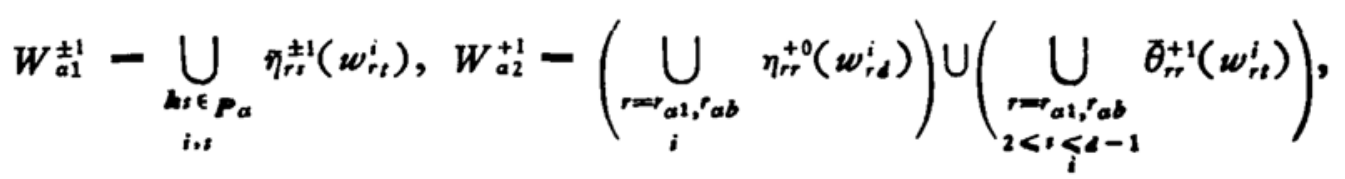

$$
\begin{aligned}
& W_{a 2}^{-1}-\left(\bigcup_{\substack{r=r_{a 1}, r^{\prime} a b \\
i}} \eta_{r r}^{+0}\left(w_{r d}^{i}\right)\right) \cup\left(\bigcup_{\substack{r=r_{a 1}, r_{a b} \\
2<i<i d-1}} \bar{\theta}_{r r}^{-1}\left(w_{r t}^{i}\right)\right), \\
& \left.W_{a}^{ \pm 1}-W_{a 1}^{ \pm 1} \cup W_{a 2}^{ \pm 1}, D^{ \pm 1}-\left(\bigcup_{,} D_{r}^{ \pm 1}-W_{a 2}^{ \pm 1}\right) \cup\left(\bigcup_{i, 1} \eta_{r_{a b^{\prime} a 1}^{ \pm 1}}^{ \pm w_{r_{a b}, t}^{t}}\right)\right),
\end{aligned}
$$


不难看出， $Y_{a}^{ \pm 1} 、 W_{c}^{ \pm 1}$ 均为偶圈之并. 这样得到 $8 \mathrm{a}$ 个 1-因子. 余下的两个 1-因子恰为 $D^{ \pm 1}$ 。 综上可知, $L^{2}(G)$ 可 1-因子分解,从而定理 1 和定理 2 成立.

\section{考文献}

[1] Fioriai, S. \& Wilson, R. J., Selected Topics in Graph Theory, Academic Press, London and New York, 1979, 103-126.

[2] Harary, F. \& Robinson, R. W., J. Graph Theory, 9 (1985), 67-86.

[3] Harary, F., Graph Theory, Addison-Wesley, Reading, Mass., 1969.

[4] Jaeger, F., Discrete Math., 9 (1974), 161-172.

[5] Zhao Guang-fu, J. Graph Theory, 12 (1988), 285-294. 